\title{
Biologie et action toxique du plutonium et des transplutoniens $\left({ }^{*}\right)$
}

\author{
J.-C. NÉNOT, J. LAFUMA (**) \\ (Manuscrit reçu le 6 avril 1976)
}

\begin{abstract}
RÉSUMÉ
Le métabolisme du plutonium et des transplutoniens est analysé en détail aussi bien dans le cas d'inhalation que dans celui d'une blessure contaminée et les différentes méthodes thérapeutiques sont exposées. Ce que l'on sait de l'action toxique du plutonium est discuté ainsi que les problèmes spécifiques du " point chaud » et du mécanisme de l'action des faibles doses.

Enfin, la méthode utilisée pour fixer les limites de sécurité et les techniques de surveillance des travailleurs sont présentées.
\end{abstract}

\begin{abstract}
A detailed analysis of plutonium and transplutonium elements is made both in the case of inhalation and contaminated wounds, and the various therapeutic procedures are given. The present state of knowledge on the toxicity of plutonium is discussed as well as the specific problems of "hot spots" and the mechanism of the effect of low level doses. Finally, the approach to the setting of safety standards and the methods of workers' surveillance are presented.
\end{abstract}

$\left(^{*}\right)$ Ce texte rassemble les deux conférences présentées par MM. Lafuma et Nénot lors de la journée d'information organisée conjointement par la Société française d'Énergie nucléaire, la Section française de l'American Nuclear Society et la Société française de Radioprotection, le 11 mars 1976 à l'École centrale des Arts et Manufactures à ChâtenayMalabry, sur le thème : "Toxicité et écologie du plutonium et des transplutoniens «.

(**) Commissariat à l'Énergie atomique, Département de Protection, B.P. $n^{\circ} 6,92260$ Fontenay-aux-Roses.

RADIOPROTECTION, VOL. $11-\mathrm{N}^{\circ} 3$ 


\section{INTRODUCTION}

L'étude de la biologie du plutonium et des transplutoniens a un but réaliste : la meilleure connaissance du métabolisme de l'élément et des doses qu'il délivre à l'organisme doit conduire à la codification de thérapeutiques applicables en cas de contamination de personnes; celle des effets toxiques, au premier rang desquels se situent les effets à long terme, doit conduire d l'établissement des limites acceptables pour les travailleurs et la population. Une telle étude ne peut être que scientifique, basée sur des faits précis, ou, à défaut, sur des hypothèses réalistes. Il faut être conscient que les connaissances acquises sur le plutonium sont extrêmement vastes, surtout si on les compare à celles concernant des toxiques plus conventionnels.

Les nombreuses recherches sur la biologie et les effets du plutonium et des transplutoniens, le souci des chercheurs d'étudier les effets du toxique avant son utilisation à grande échelle peuvent expliquer en partie l'émotion et la passion qui alimentent parfois des polémiques de nature pseudo-scientifique. Il n'en reste pas moins vrai qu'une meilleure maitrise du plutonium dans son ensemble passe par la plus grande connaissance possible de ses propriétés biologiques.

\section{MÉTABOLISME}

Dès l'année 1944, des recherches axées sur le devenir du plutonium 239 dans l'organisme vivant furent entreprises à l'Université de Berkeley. Rapidement les recherches se sont développées, tant à l'Université de Chicago qu'aux Laboratoires de Los Alamos et à l'Université de Rochester. Il a fallu environ 5 ans pour que se dégagent les grandes lignes concernant le comportement biologique du plutonium chez l'animal. Ces études furent motivées par le souci de la protection des travailleurs, car de très nombreuses inconnues existaient; entre autres, on ne savait pas quel facteur de gravité attribuer aux quantités de plutonium même infimes que quelques travailleurs rejetaient journellement dans leurs urines. C'est pourquoi en 1945 et 1946 des recherches curent entreprises pour essayer de corréler les quantités de plutonium excrétées aux quantités de plutonium présentes dans l'organisme.

\subsection{DONNÉES humaines}

C'est ainsi que des études sur l'homme furent menées conjointement aux Laboratoires de Los Alamos, aux Universités de Rochester et de Berkeley. Le problème posé était apparamment simple : quelle signification fallait-il donner à une certaine quantité de plutonium mesurée dans l'urine ? Pour répondre à cette question, des activités connues furent injectées à des malades condamnés à brève échéance par la médecine, et les quantités excrétées furent mesurées. Bien qu'en nombre limité, ces données sont importantes car ce sont les seules 
chez l'homme pour lesquelles on connaît la quantité administrée. Les résultats d'excrétion sont encore actuellement à la base des estimations des charges corporelles en plutonium à partir des données d'excrétion, par exemple pour les travailleurs qui incorporent accidentellement du plutonium.

D'autres données proviennent des quelques accidents survenus dans l'industrie du plutonium; ces données ne sont guère utilisables pour la validation du modèle animal puisque la donnée principale - quantification exacte de l'apport - manque. Par contre, elles sont essentielles pour l'étude des effets.

\subsection{EXPÉRIMENTATION ANIMALE}

Peu de toxiques peuvent s'enorgueillir d'avoir suscité autant de recherches biologiques, qu'elles relèvent du domaine métabolique, toxicologique ou thérapeutique. Le plutonium a sans doute été le premier toxique dont les premiers milligrammes produits aient été destinés à la recherche biologique, donc à la protection; il est plus habituel de voir ce genre d'études suivre, et souvent de loin, la production industrielle d'un toxique. Les données expérimentales s'accumulent donc depuis maintenant une trentaine d'années et viennent d'horizons divers : plusieurs équipes aux États-Unis travaillent sur des espèces différentes, comme les rongeurs et les chiens, accessoirement sur les singes, ainsi que plusieurs laboratoires russes; les chercheurs européens, essentiellement en France, ont mis leurs efforts de recherche sur les petits animaux; ce choix leur a permis de combler le retard initial pris dans les recherches, l'utilisation de gros animaux, singes par exemple, ne servant qu'à vérifier l'extrapolation possible de résultats ou à pratiquer des expériences impossibles sur des animaux de petite taille.

\section{Rapport entre la nature physicochimique du plutonium et son comportement biologique}

Le métabolisme du plutonium est sous la dépendance directe de sa forme physicochimique. Les propriétés chimiques de cet élément sont particulièrement complexes. De plus, en milieu biologique, le plutonium ne peut être transporté que lié à des composés naturels réalisant ainsi des complexes physiologiques; ces composés sont soit des protéines ou autres macromolécules (sang) soit des constituants cellulaires de faible poids moléculaire, comme des citrates ou autres anions organiques ou comme des acides aminés (tissus). La protéine de transport plasmatique a été identifiée comme étant la sidérophiline, servant normalement au transport du fer dans l'organisme. L'existence de plutonium soluble, sous forme ionique libre, est impossible en milieu vivant car incompatible avec ses propriétés chimiques; il ne peut, en effet exister en solution qu'à des $\mathrm{pH}$ très acides (grossièrement inférieurs à 1); il précipite sous forme d'hydroxyde dès que le $\mathrm{pH}$ s'élève; cette dernière condition est réalisée lors de l'introduction directe dans un tissu, dans le cas d'un accident par blessure par exemple. Il convient d'ajouter que le plutonium peut exister en solution sous des formes extrêmement variables, 
avec des états de valence allant de $3+$ à $6+$. La forme la plus fréquemment rencontrée en milieu biologique est la forme $4+$; elle correspond à la forme complexée qui est, en fait, la destinée biochimique du plutonium.

Le composé le plus préoccupant du plutonium 239 est sans doute sa forme oxydée, $\mathrm{PuO}_{2}$, puisque ses propriétés en font un combustible de choix. Le comportement de l'oxyde de plutonium depuis son incorporation dans le milieu biologique varie en fonction de facteurs très différents comme la composition exacte, la taille et la forme de la particule, son âge, la température à laquelle la calcination a eu lieu.

Il faut souligner les difficultés rencontrées dans l'intercomparaison des résultats, qu'ils soient tirés de l'expérimentation animale ou d'analyses de cas humains; elles sont en rapport direct avec la difficulté de reproductibilité des conditions, qui ne fait que s'ajouter aux variations individuelles qui sont la règle en matière de biologie.

\section{Voies d'entrée dans l'organisme}

Comme tout agent contaminant, le plutonium peut s'introduire dans l'organisme par trois voies différentes : le plutonium qui se trouverait dans l'alimentation peut être ingéré puis absorbé au niveau du tube digestif; le plutonium qui serait dispersé dans l'atmosphère peut soit se déposer sur la peau et y être absorbé, soit être inhalé et retenu dans l'arbre respiratoire et secondairement absorbé à ce niveau; l'introduction par blessure demeure, avec l'inhalation, l'apanage des professionnels.

Par voie digestive, le plutonium n'est pratiquement pas absorbé : une solution nitrique acide $1 / 100 \mathrm{~N}$, soluble, ne donne lieu qu'à $0,002 \mathrm{p}$. cent d'absorption chez le rat et le porc. Ceci est en rapport avec l'hydrolyse brutale qui se produit dès l'arrivée du plutonium dans le duodénum qui a un $\mathrm{pH}$ alcalin. Les formes oxydées sont encore moins absorbées : on donne des chiffres de l'ordre de grandeur de $0,0001 \mathrm{p}$. cent. L'absorption peut, cependant, être plus élevée pour des formes plus rarement rencontrées, comme le citrate de plutonium (jusqu'à $1 \mathrm{p}$. cent).

La voie d'entrée au travers de la peau saine ne représente pas, non plus, une voie de contamination importante; il n'a pas été clairement prouvé qu'elle existait réellement.

L'inhalation représente certainement la façon la plus probable dont l'homme risque d'absorber des quantités significatives de plutonium. La fraction de plutonium inhalé qui est retenu dans le poumon dépend des propriétés physicochimiques du composé ainsi que de la fonction respiratoire de l'individu. Schématiquement, la fraction déposée dans le poumon profond varie en sens inverse du diamètre de la particule, alors que celle déposée dans les voies aériennes supérieures varie dans le même sens. C'est la première fraction qu'il est important de comptabiliser; elle conditionne, en effet, les dépôts relatifs dans le poumon et dans les différents organes après passage au travers de la paroi alvéolaire. La vitesse de ce passage est très variable, toute la gamme existe, de la vitesse quasi nulle à des vitesses correspondant à des périodes biologiques de quelques semaines. 
On retrouve les mêmes variations de la cinétique dans le cas des contaminations par blessure. L'étude des blessures contaminées présente une difficulté expérimentale : la reproductibilité est illusoire, et de ce fait, les paramètres du modèle métabolique difficiles à chiffrer. La plupart des expérimentateurs ont donc choisi l'injection intraveineuse comme modèle, dont le moins que l'on puisse dire est qu'elle manque de réalisme. De plus, comme le plutonium injecté directement dans la circulation s'hydrolyse avec formation de gros colloïdes entraînant des conséquences immédiatement fâcheuses sur le plan physiologique, il a été nécessaire de s'adresser à des composés stables à $\mathrm{pH}$ neutre. C'est ainsi que la plupart des expériences furent menées avec du citrate de plutonium, le métabolisme était alors plus calqué sur celui du citrate que sur celui du plutonium, étant donnée la stabilité assez grande du complexe.

\section{Transfert, incorporation, élimination naturelle}

Après s'être déposé au niveau de la porte d'entrée - tube digestif, peau, poumons ou blessure - le plutonium passe dans la circulation sanguine. Alors que la cinétique de passage varie énormément d'un composé à un autre, par contre le mode de fixation dans l'organisme du plutonium véhiculé par le sang ne dépend pratiquement plus ni du lieu d'origine, ni de la forme sous laquelle il a été véhiculé; seule, diffère la répartition relative entre les différents organes. Cette répartition est donc, dans l'ensemble, stéréotypée.

Le poumon représente un cas particulier, car il existe un processus naturel d'épuration qui débarrasse l'arbre respiratoire de la plus grande partie des particules inhalées; cette épuration s'effectue selon deux modes :

- un mode absolu, aboutissant au rejet du toxique par remontée le long de l'arbre respiratoire jusqu'au carrefour pharyngé, puis passage secondaire dans le tube digestif avec excrétion quasi totale dans les excreta; ce mode d'épuration a deux composantes : l'une rapide, purement mécanique, débarrassant les voies aériennes supérieures, trachée et bronches, avec des périodes biologiques de l'ordre de grandeur du jour, l'autre lente, associant des processus cellulaires et mécaniques, débarrassant le poumon profond, alvéolaire, avec des périodes biologiques supérieures au mois pour les formes les plus solubles de plutonium et à l'année pour l'oxyde de plutonium 239 . La cellule responsable de cette épuration alvéolaire est le macrophage alvéolaire, qui après avoir phagocyté une ou plusieurs particules, meurt sous l'effet de l'irradiation $\alpha$ et est éliminée du poumon.

- un mode relatif, par passages vers les ganglions lymphatiques thoraciques dans lesquels le plutonium est retenu pratiquement dans sa totalité; ainsi, pour l'oxyde de plutonium 239, environ $10 \mathrm{p}$. cent seulement de la quantité déposée dans les ganglions bronchopulmonaires migrent vers le sang, donc vers les organes avec une période biologique très supérieure à l'année.

C'est la fraction disponible qui migre vers les territoires de rétention avec des vitesses en rapport avec l'état physicochimique du plutonium. La

vOL. $11-\mathrm{N}^{\circ} 3$ 
combinaison des vitesses de ces modes d'épuration du poumon conditionne l'importance du dépôt secondaire dans les organes.

Le passage du plutonium vers les tissus se fait aussi suivant des lois exponentielles avec, semble-t-il, plusieurs composantes dont les coefficients augmentent au fur et à mesure que l'on s'éloigne de la contamination initiale. Schématiquement et en se basant sur l'expérimentation animale, pratiquée soit sur le rat, soit sur le chien Beagle, le plutonium inhalé sous forme soluble est épuré en majeure partie pendant les deux premières années et se fixe principalement au niveau du foie et du squelette, alors que le plutonium sous forme insoluble est retenu beaucoup plus longtemps et est bloqué en très grande partie par les ganglions lymphatiques drainant les territoires pulmonaires; par exemple, 10 ans après l'inhalation d'oxyde de plutonium 239 chez des chiens, 20 p. cent seulement sont épurés, sur les 80 p. cent restant, 10 p. cent demeurent dans le poumon, 40 p. cent sont fixés dans les ganglions thoraciques et le reste dans le foie et l'os. Dans les ganglions, le plutonium se concentre surtout au niveau des sinus médullaires et peu dans les centres germinatifs; la concentration hétérogène dans les ganglions a été vérifiée chez l'homme.

Dans le cas d'une contamination par blessure, il est tout à fait impossible d'évaluer a priori la quantité qui migrera dans l'organisme puisqu'elle dépend, de surcroît, des conditions inhérentes à la blessure, tels que localisation, profondeur, degré d'attrition, etc. C'est pourquoi le modèle expérimental idéal n'existe pas, et le moins mauvais, parce que reproductible, est représenté par l'injection intramusculaire. Dans le cas d'injection de plutonium sous forme de sel soluble, faiblement ou non complexé, nitrate par exemple, la quantité migrant dans l'organisme peut atteindre plusieurs pour cent, ce qui peut finalement représenter une activité non négligeable au niveau du squelette. Mais surtout, cette fraction ne migre pas avec une vitesse constante : elle est d'autant plus grande que l'on est plus proche de la contamination; ceci est dû à la compétition entre les phénomènes d'hydrolyse locale aboutissant à la formation in situ d'hydroxydes très lentement dégradables et les phénomènes de chélation naturelle avec les transporteurs plasmatiques. Ce phénomène n'est pas propre aux contaminations par blessure mais il y apparaît plus nettement; il en découle une conséquence importante et immédiate pour le thérapeute : mise en œuvre d'urgence du traitement de la contamination.

Ainsi, la migration du plutonium aboutit, par suite des lois d'équilibre biochimique, au dépôt dans les organes, certains étant préférentiels comme le foie et le squelette. Au niveau du foie, la fraction retenue et les vitesses d'épuration dépendent de la forme du plutonium et de l'espèce contaminée. Ainsi, le dépôt hépatique est d'autant plus important après une intraveineuse que le degré de polymérisation du plutonium injecté est élevé, et la période biologique peut varier d'un facteur $4-$ de 3 semaines à 3 mois. D'une façon générale, les rongeurs vident leur foie assez rapidement, à l'inverse des chiens et des primates, espèce humaine comprise. Ainsi, en extrapolant et en comparant les résultats de nombreuses espèces, une période biologique hépatique 
de 40 ans paraît réaliste pour l'homme. Dans l'organe, le plutonium lié avec la sidérophiline se fixe primitivement dans les hépatocytes, selon une répartition régulière; l'accumulation est nette au niveau des lysosomes. Petit à petit se fait une agrégation dans les cellules de Küpffer, dépendant du système réticulo-endothélial, en même temps que les cellules hépatiques proprement dites régénèrent. L'importance de la contamination, donc du niveau d'irradiation de l'organe, conditionne aussi l'épuration du plutonium déposé dans le foie.

Au niveau du squelette, le plutonium qui arrive lié à la sidérophiline est déposé préférentiellement sur la surface endostéale de l'os; cette localisation est importante car elle constitue une position péjorative pour la santé de l'individu : il semble bien que ce soit une position privilégiée pour l'induction des cancers osseux $(c f$. $\S 4)$. En fonction du taux de croissance et de renouvellement de cette partie du squelette, on peut assister dans des proportions variées à un enfouissement progressif dans l'os profond; une concentration dans les ostéoclastes qui ont la charge de la résorption osseuse est aussi possible. Une migration vers la moelle osseuse s'opère lentement, grâce aux macrophages qui se saisissent du plutonium libéré en surface. Toutes ces modifications morphologiques tendent à faire perdre au plutonium une partie de sa nocivité et la période que l'on pourrait qualifier de critique est, peut-être, plus limitée dans le temps que la durée de séjour du plutonium. Ce temps de séjour est toujours très long, les périodes biologiques étant toujours de l'ordre de grandeur de la durée de vie de l'animal considéré : un an pour la souris, plus de 10 ans pour le chien, elle est estimée à environ 100 ans pour le squelette de l'homme.

Il existe d'autres territoires de rétention du plutonium mais dans des proportions moindres, bien que les activités spécifiques puissent, de par le faible poids des organes en cause, être très élevées; on retrouve, ainsi, des quantités non négligeables au niveau d'organes tels que la rate, les testicules, la thyroïde. Chez l'homme, on estime que $10 \mathrm{p}$. cent environ du plutonium fixé par l'organisme, porte d'entrée exceptée, se répartissent dans les territoires extra-hépatique et extra-osseux.

Malgré ces rétentions très longues, il existe une élimination naturelle, qui ne concerne jamais qu'une faible fraction de plutonium transportée par le sang. C'est, cependant, sur l'élimination urinaire que l'on possède le plus de résultats chiffrés chez l'homme, parce que l'analyse des excreta constitue la méthode la plus sensible et, dans de nombreux cas, la seule méthode pour mettre en évidence la présence de plutonium dans l'organisme. De nombreuses équations ont été proposées liant l'excrétion urinaire et le dépôt initial de plutonium, mais en aucun cas on ne peut considérer que l'estimation de la charge corporelle à partir de l'élimination urinaire soit très précise. Très grossièrement, l'excrétion totale représente environ $5 \mathrm{p}$. cent les trois premières semaines après une injection unique, $10 \mathrm{p}$. cent les deux premières années, et un peu plus de $20 \mathrm{p}$. cent après 40 ans.

En conclusion, toute contamination par le plutonium, en plus du dépôt sur la porte d'entrée, qui, pour un organe comme le poumon, constitue un

vol. $11-\mathrm{N}^{\circ} 3$ 
risque potentiel lié à la dose délivrée, aboutit à la localisation de l'élément dans des organes - essentiellement le tissu osseux - dans lesquels il séjourne des temps extrêmement longs.

\subsection{Comparaison avec D'Autres actinides Émetteurs alpha}

Il s'agit essentiellement de l'isotope 238 du plutonium et d'éléments de la série des transplutoniens : américium 241 , curium 242 et 244 , californium 252, einsteinium 253, par exemple. La comparaison de ces divers éléments est des plus intéressantes car elle permet de relier certaines caractéristiques propres à l'élément aux caractéristiques du comportement biolologique et à l'expliquer; par exemple :

- importance de la masse pondérale en cause, grâce à la comparaison des plutonium 238 et 239 ou des curium 242 et 244 . Ainsi, après une injection intramusculaire, le plutonium 239 s'individualise des autres actinides - y compris le plutonium 238 - par la lenteur de sa migration; à activité égale, l'isotope 239 du plutonium représente de loin la masse la plus élevée;

- importance de l'état de valence, par comparaison du plutonium, valence $4+$, avec les transplutoniens, valence $3+$; c'est après une inhalation que s'extériorise le mieux l'influence de la physicochimie de l'élément : inhalés sous la forme de sels nitriques, les deux plutonium 238 et 239 sont bien groupés alors que les transplutoniens s'épurent beaucoup plus vite;

- importance du débit de dose délivré à un organe sur la cinétique de ses rétentions par comparaison d'un émetteur à vie courte, comme le curium 242, avec un émetteur à vie assez longue, comme l'américium 241, etc.

D'une façon générale, l'état de valence $3+$ confère aux transplutoniens une solubilité in vivo très supérieure à celle du plutonium, ce qui se traduit par des vitesses de migration à partir de la porte d'entrée beaucoup plus grandes. Ceci est en rapport avec un $\mathrm{pH}$ d'hydrolyse beaucoup plus élevé que dans le cas du plutonium et se traduit par des dépôts beaucoup plus homogènes au niveau des organes, au premier rang desquels se situe le poumon; ainsi, l'inhalation de curium ou d'américium en solution aboutit au dépôt de l'élément sous forme monomérique, alors que le plutonium inhalé dans les mêmes conditions donne un dépôt hétérogène sous forme d'hydroxydes apparaissant sur les autoradiographies comme des étoiles en forme d'oursins. Schématiquement, plus on avance dans la chaîne des transplutoniens, plus la solubilité dans l'organisme augmente. Le résultat pratique consiste, parallèlement à une épuration rapide de la porte d'entrée, en une charge osseuse très rapide : ainsi, le dépôt dans le squelette de californium 252 atteint la moitié de son maximum seulement un jour après la contamination par intramusculaire; le maximum est atteint en 3 semaines. Ces valeurs sont très éloignées de celles obtenues avec le plutonium 239. Une fois passées les premières semaines, on ne constate plus de différences notables entre les dépôts osseux des transplutoniens de valence $3+$, et leur cinétique variable d'un isotope à un autre. 
Cette diffusion importante a pour conséquence le dépôt de l'élément dans une multitude d'organes et tissus qui semblaient en être exempts avec le plutonium; ce dépôt est évidemment moins élevé que pour le squelette, mais après une contamination par un transplutonien, on trouve toujours dans la majorité des tissus de l'organisme une activité alpha mesurable, fixée. Ceci est spécialement net au niveau du rein, où cela est peut-être tout simplement dû à l'élimination urinaire relativement élevée de ces éléments, comparée au plutonium.

Un autre point mérite d'être signalé : alors que le plutonium sous forme oxydée est considéré comme insoluble (et certains oxydes récents calcinés à très haute température présentent réellement un très haut degré d'insolubilité dans l'organisme vivant), les transplutoniens, par contre, échappent à cette règle. Il existe toujours une certaine dégradation et certains oxydes d'américium et de curium ont même prouvé être plus rapidement solubilisés par la matière vivante que des sels solubles secondairement hydrolysés.

Ainsi, les deux caractères fondamentaux qui différencient les transplutoniens du plutonium 239 sont :

- absorption rapide;

- grande diffusion dans l'organisme;

et les conséquences qui en découlent sont d'ordre thérapeutique et pathologique.

\section{POSSIBILITÉS THÉRAPEUTIQUES}

La pierre d'achoppement du traitement des contaminations internes est la lenteur de l'élimination, quand elle existe, des radionucléides quand ceux-ci sont fixés dans l'organe critique. C'est pourquoi le traitement d'urgence qui agit au niveau de la porte d'entrée ou du véhicule sanguin revêt une telle importance; ce traitement n'est applicable que dans le cas d'accidents ou d'incidents lorsque la contamination est reconnue.

Le traitement radical est évidemment l'ablation du contaminant et des tissus contaminés avant qu'une migration ait pu se produire. Une telle éventualité est purement théorique et même quand l'ablation de l'agent contaminant est possible (aiguille de plutonium dans une main, par exemple), il faut faire appel à la chimiothérapie, qui, dans ce cas précis, servira de couverture et empêchera tout dépôt dans les organes de rétention. En effet, il existe des drogues chélatrices qui forment, avec le plutonium, les éléments de valence $3+$ (série des lanthanides et transplutoniens) et certains métaux, des complexes extrêmement stables, totalement et rapidement éliminés de l'organisme par voie urinaire. Celle qui combine la meilleure efficacité et la moindre toxicité est l'acide diéthylènetriaminopentaacétique (DTPA). Malheureusement, ce produit n'agit que sur le plutonium soluble, c'est-à-dire lié à un chélateur naturel et uniquement dans les compartiments extracel-

voL. $11-\mathrm{N}^{\circ} 3$ 
lulaires. C'est dire que son action, dès lors que le contaminant est sous forme insoluble comme l'oxyde de plutonium ou qu'il est fixé dans le squelette, est pratiquement nulle. Une légère décontamination de l'os par le DTPA a été mise en évidence chez le rat pour l'américium, le curium et le plutonium 238, mais rien ne prouve qu'elle existe chez l'homme et surtout qu'elle se poursuive très longtemps. De toute façon, elle ne jouerait que sur des fractions faibles, la période biologique de l'élément dans l'os n'étant que très modérément accélérée. Le traitement par DTPA doit être institué d'autant plus rapidement que l'élément toxique migre rapidement. C'est le cas des transplutoniens et quelquefois de l'isotope 238 du plutonium. La règle d'or du traitement est donc, avant tout, la rapidité de sa mise en œuvre. Ce premier geste thérapeutique donne le temps nécessaire pour procéder aux premiers examens indispensables et pour décider de la conduite ultérieure du traitement.

Le DTPA est utilisé chez l'homme depuis une quinzaine d'années pour des contaminations ou suspicions de contamination; aucune pathologie secondaire n'a été observée sur les centaines de travailleurs qui ont subi ce traitement.

Dans le cas où l'élément est sous forme insoluble - l'exemple représentatif est l'oxyde de plutonium puisque les formes oxydées des transplutoniens se sont avérées être biologiquement solubles - le DTPA est inopérant. La seule possibilité offerte est alors l'ablation du radiotoxique : dans le cas d'une blessure, c'est l'exérèse chirurgicale; dans le cas d'une contamination par inhalation, c'est le lavage pulmonaire; cette dernière technique, consiste à irriguer le poumon - lobe par lobe - à l'aide d'un soluté physiologique, qui entraîne ainsi les particules toxiques. Cette technique est utilisée en clinique humaine pour de nombreuses affections obstructives des poumons et n'est pas réservée aux seuls radionucléides; elle reste, cependant, une thérapeutique d'exception. Pour le plutonium, elle a fait ses preuves expérimentales (efficacité de 50 à 90 p. cent) et humaine (un cas aux U.S.A.). L'inconvénient majeur du lavage réside, non pas dans ses risques qui sont extrêmement réduits, mais dans la nécessité de le répéter plusieurs fois, imposant ainsi au contaminé, en bonne santé par ailleurs, une lourde sujétion.

Il faut souligner qu'il est toujours observé chez les animaux d'expérience une réduction des effets nocifs - raccourcissement de la durée de vie, cancers pulmonaires, osseux ou autres - en rapport direct avec la réduction de la charge en plutonium obtenue grâce au traitement.

\section{ACTION TOXIQUE DU PLUTONIUM ET DES TRANSPLUTONIENS}

\subsection{OBSERVATIONS hUMAINES}

Malgré leur toxicité élevée, on ne connaît aujourd'hui aucun cas d'être humain ayant présenté des troubles dus à une contamination par ces éléments. Toutes les données sur l'action toxique proviennent de l'expérimentation animale. 
Un certain nombre de travailleurs ayant manipulé du plutonium sont actuellement suivis médicalement [1] :

- le premier groupe - le plus ancien - est composé de 29 personnes ayant participé au projet Manhattan. Leurs contaminations pulmonaires remontent aux années 1944 et 1945. Elles étaient comprises à l'origine entre 10 et $200 \mathrm{nCi}$ de ${ }^{239} \mathrm{Pu}$, soit de 1 à 15 fois la charge maximale admissible dans l'organe;

- le deuxième groupe est composé de 25 personnes contaminées en 1965 à Rocky-Flat. Les contaminations pulmonaires étaient, à cette époque, comprises entre 1 et 10 fois la charge maximale admissible dans l'organe;

- aux U.S.A., encore, il existe un groupe de personnes qui ont manipulé du plutonium et qui sont contaminées à des niveaux inférieurs à la charge maximale admissible pulmonaire;

- on connaît, enfin, des cas de personnes ayant eu une blessure contaminée par le plutonium 239. L'une d'entre elles a été accidentée en France, en 1962 [2]. La quantité de plutonium incorporée dans la blessure était, à l'origine, de l'ordre du milligramme. Une thérapeutique de décontamination a permis de diminuer, mais sans le ramener à zéro, le niveau de la contamination de la main et celui de la contamination interne. On n'a observé, à ce jour, aucune lésion chez cette personne malgré les niveaux très élevés de la contamination;

- un autre cas bien connu est celui du " soi-disant cancer » dû à une seule particule de plutonium incorporée dans une blessure [3]. Il s'agit d'une particule de plutonium qui a été excisée d'une blessure 4 ans après l'accident. La dose locale totale était de 75 millions de rads. L'examen anatomo-pathologique n'a pas révélé la présence d'un cancer mais d'une lésion semblable à celles que l'on observe dans les modifications pré-cancéreuses de l'épiderme. Mais ce type de lésion n'étant pas spécifique des étapes pré-cancéreuses, on ne peut que se poser des questions sur ce qu'aurait pu être son devenir.

Sur les travailleurs contaminés par le plutonium 239, on a pratiqué des recherches d'aberrations chromosomiques. Les résultats en sont contradictoires. Aucune modification n'a été observée chez les travailleurs du projet Manhattan, alors qu'on en a trouvé parmi ceux de Rocky-Flat. Le petit nombre d'examens, chez les sujets contaminés aussi bien que chez les témoins, rend difficile l'interprétation, d'autant plus que des études expérimentales sur l'animal n'ont pas montré d'aberration pour des doses inférieures à 1000 rads $\alpha$.

\subsection{DONNÉES EXPÉRIMENTALES}

Celles-ci sont de deux types : contamination par voie intraveineuse pour étudier l'action toxique au niveau du squelette et contamination par voie respiratoire pour suivre les conditions réelles. 


\section{Injections intraveineuses}

On possède de nombreuses données sur l'action de l'injection intraveineuse de citrate de plutonium ou de transplutoniens. Les études métaboliques ont montré le côté totalement artificiel de cette approche du problème. Néanmoins, ces études ont permis de comparer l'action toxique du citrate de plutonium à celle du radium ou du strontium 90 injectés par la même voie.

Deux expériences importantes ont été faites avec des chiens Beagle. La première, qui est terminée, a utilisé des activités injectées comprises entre 3000 et $16 \mathrm{nCi}$ par kilogramme d'animal. Au niveau le plus bas, on a observé 30 p. cent de cancers osseux.

La deuxième expérience, qui est en cours, porte sur des activités plus faibles descendant jusqu'à $0,6 \mathrm{nCi} . \mathrm{kg}^{-1}$, valeur qui est celle de la charge maximale admissible du squelette. Il faudra attendre encore plusieurs années pour avoir les résultats de cette expérience.

Un certain nombre d'expériences ont été pratiquées sur des rongeurs. Elles ont montré que pour les mêmes doses totales cumulées, on avait des fréquences de cancers osseux comparables à celles observées avec les chiens.

\section{Inhalation}

Comme pour les injections intraveineuses, il existe deux groupes de chiens Beagle contaminés avec de l'oxyde de ${ }^{239} \mathrm{Pu}$ [1]. Le premier groupe a reçu des activités entraînant des doses supérieures à 1000 rads. Au niveau le plus faible ( $7 \mathrm{nCi} \cdot \mathrm{g}^{-1}$ de poumon) on a observé 7 cancers sur 8 animaux. Le deuxième groupe, actuellement en expérimentation, a reçu des activités plus faibles, descendant jusqu'à la charge maximale admissible humaine. D'autres groupes de chiens sont actuellement suivis après contamination par le nitrate de ${ }^{239} \mathrm{Pu}$, l'oxyde et le nitrate de ${ }^{238} \mathrm{Pu}$, de ${ }^{241} \mathrm{Am}$ et de ${ }^{244} \mathrm{Cm}$.

De nombreuses données ont été obtenues, avec des rongeurs, aux U.S.A., en U.R.S.S. et en France. Elles ont montré l'apparition de cancers pulmonaires pour des activités déposées supérieures à $1 \mathrm{nCi} \cdot \mathrm{g}^{-1}$ (60 fois la charge maximale admissible chez l'homme).

En France, on a comparé l'action toxique du plutonium 239, du plutonium 238, de l'américium 241 et du curium 244 sous deux formes chimiques : solutions et oxydes. Les résultats ont montré que la fréquence des cancers n'était pas plus élevée lorsque le radionucléide était sous forme particulaire (point chaud). Ces expériences ont fourni des données suffisantes pour des doses supérieures à 100 rads $\alpha$ [4].

\section{Les problèmes actuels en toxicologie expérimentale du plutonium et des transplutoniens}

Aujourd'hui deux problèmes importants restent posés :

- l'action d'une dose locale élevée (le point chaud);

- la forme de la relation dose-effet aux faibles niveaux de dose. 
Les deux problèmes ne sont que deux aspects différents d'une même conception de la carcinogenèse radio-induite. Le mécanisme envisagé est grossièrement le suivant : l'irradiation modifie les molécules, ce qui, ensuite, entraîne une mutation cellulaire qui suffit pour induire un cancer. Ce type de mécanisme est probablement vrai pour les cancers provoqués par des virus; il existe, cependant, des arguments contraires en ce qui concerne les cancers radio-induits.

Tout d'abord, la succession des trois étapes n'a jamais été formellement prouvée par l'expérimentation. Ensuite, si à l'échelle cellulaire, les cinétiques sont comparables, on observe malgré cela des cancers à la fin de la vie de toutes les espèces. Cette simple observation prouve l'existence d'un mécanisme fondamental au niveau de l'organisme entier. De plus, si l'on donne aux différentes espèces, les mêmes doses cumulées pendant les mêmes fractions de leurs durée de vie, on observe les mêmes fréquences des mêmes cancers [5].

Enfin, si l'induction des cancers n'était due qu'à un phénomène probabiliste cellulaire, proportionnel à la dose, on devrait, en diminuant celle-ci, n'observer qu'une diminution de la fréquence, sans modification de la répartition dans le temps. En réalité, la diminution de la dose allonge l'intervalle de temps auquel apparaissaient les tumeurs. Plus le temps s'allonge, et plus l'animal a de chances de mourir d'autres causes. C'est, en réalité, l'allongement du temps de latence qui est la cause de la diminution de la fréquence quand la dose diminue.

\section{Le problème du point chaud}

Ce problème a été abordé de deux façons différentes en France et aux U.S.A. En France, on a comparé des émetteurs $\alpha$ ayant dans le poumon des répartitions très différentes; aux U.S.A. on a injecté, pour qu'elles se bloquent dans les poumons, des billes d'oxyde de zirconium marquées avec différentes activités de ${ }^{238} \mathrm{Pu}$, ceci pour simuler des points chauds, plus ou moins actifs et en nombre connu. Les expériences françaises ont montré qu'une répartition hétérogène d'une activité donnée dans le poumon n'était pas plus cancérigène que la même activité répartie de façon homogène. Par contre, l'action létale était beaucoup plus importante dans le deuxième cas.

Les expériences américaines ont montré qu'au lieu des 45000 cancers calculés par la méthode de TAMPLIN et COCHRAN, on en avait observé seulement 4. Les deux expériences qui se recoupent montrent que la théorie du point chaud est fausse.

\section{L'effet des faibles doses}

Pour l'expérimentateur, les faibles doses sont situées dans une gamme allant de 1 à 10 rads $\alpha$ délivrés pendant la durée de la vie. En comparant les données expérimentales obtenues avec les émetteurs $\alpha$ et les données humaines tirées de l'observation des fumeurs, on peut dire que ces doses correspondraient à une consommation humaine comprise entre 50 et 500 paquets de cigarettes.

VOL. $11-\mathrm{N}^{0} 3$ 
A de tels niveaux de dose, l'observation directe des effets ne serait possible qu'en utilisant un nombre d'animaux très élevé, très supérieur aux possibilités de n'importe quel laboratoire. Une seule solution est envisageable : la compréhension des mécanismes qui aboutissent à l'apparition des cancers.

Au C.E.A., nous avons entrepris l'étude des phénomènes qui se produisent pendant la phase de latence $[6,7]$. Nous avons utilisé deux méthodes : l'analyse systématique d'un million de coupes prélevées sur les poumons de 2000 animaux à différents temps de la phase de latence et la greffe systématique, sur des animaux récepteurs, d'échantillons de poumons prélevés sur les animaux en phase de latence.

\section{$1^{\circ}$ Observations systématiques}

Les expériences ont montré que, dans le temps et pour toutes les lignées cellulaires étudiées, il existait toujours la même séquence : d'abord, apparition de quelques cellules isolées d'aspect normal, en des territoires où elles ne devraient pas se trouver, ensuite augmentation du nombre de ces cellules qui envahissent une petite zone de l'organe (adénomatose ou métaplasie), puis organisation de ces cellules en tumeurs qui n'ont pas de caractère de malignité (adénone), enfin modification de la structure de ces tumeurs et apparition de caractères malins (cancers). Cette séquence est continue et il n'existe pas de frontière nette entre les différents stades, y compris entre l'adénone et le cancer. Ce qui est important c'est que l'on retrouve les mêmes étapes chez les animaux témoins au cours de leur vieillissement [4].

Tout se passe comme si l'irradiation ne faisait qu'accélérer un phénomène dynamique préexistant dans chaque animal. Plus la dose est forte et plus l'accélération est importante, donc plus le temps de latence paraîtra bref.

\section{$2^{\circ}$ Étude des greffes de fragments de poumons}

Après contamination, des fragments de poumons en début de phase de latence ont été greffés sur des souris spéciales qui acceptaient des greffes de fragments d'organes provenant de n'importe quelle espèce [8]. En une dizaine de jours, on observe une modification très rapide de la structure du greffon qui prend un aspect morphologique pseudo-cancéreux, mais sans présenter des caractères de malignité. Là encore, les mêmes modifications sont observées sur les animaux âgés et la précocité de la transformation est liée à la dose de radionucléide inhalée par l'animal donneur. La conclusion est de nouveau que l'irradiation accélère un phénomène. Mais ce type d'expérience fournit une autre information : quel que soit le greffon prélevé sur le même poumon, on observe que l'altération porte toujours sur la même lignée cellulaire. Cette lignée sensible est liée à l'animal lui-même et varie suivant les animaux. Cette sensibilité particulière explique pourquoi le type histologique des lésions et le point de départ des cancers ne sont pas rigoureusement corrélés avec la topographie fine de la rétention des émetteurs $\alpha$.

De toutes ces expériences, on peut fournir l'interprétation suivante : tout tissu est destiné à devenir cancéreux; le temps au bout duquel ce phéno- 
mène doit se produire est, pour chacun d'entre eux, une caractéristique génétique de l'animal. Sensibilité particulière et temps de latence en l'absence de toute agression sont définis au départ. Dans une population d'animaux, la répartition des temps de latence pour un même tissu suit une fonction de répartition classique. La moyenne des temps de latence est suffisamment élevée pour que, pour un même tissu, quelques animaux seulement présentent un cancer à la fin de leur vie.

L'irradiation, quant à elle, accélère le phénomène et ce, d'autant plus que la dose a été plus élevée.

Ce que l'on peut attendre de cette conception de l'action des faibles doses:

(a) la relation dose-effet serait de type sigmoïde - ce qui a été vérifié dans de nombreux cas [4];

(b) s'ils devaient être observés, les cancers n'apparaîtraient aux faibles doses que dans les dernières tranches d'âge de la vie. Ce phénomène a été constaté dans les nombreuses enquêtes épidémiologiques consacrées aux fumeurs;

(c) on peut envisager un seuil pratique qui serait le raccourcissement de la phase de latence présentant une signification réelle pour la vie de l'individu (ce temps pourrait se chiffrer en mois);

(d) si l'on était capable d'analyser les mécanismes en jeu et de donner, pour chaque individu, la durée des différents temps de latence liée à la constitution génétique, on pourrait établir, par individu, un profil de risque et sélectionner les travailleurs soumis au risque carcinogène.

Les implications sont suffisamment importantes pour que les expérimentations des prochaines années en cancérogenèse radioactive soient consacrées à l'étude des mécanismes.

\section{LES LIMITES ACTUELLES DE SÉCURITÉ}

\section{- Charge maximale admissible du squelette : $40 \mathrm{nCi}$.}

Cette valeur a été fixée en 1949 en tenant compte d'une part, de données humaines permettant de limiter à $100 \mathrm{nCi}$ la charge maximale du squelette pour les personnes utilisant le radium 226 et, d'autre part de données expérimentales obtenues par injection intraveineuse de radium 226 ou de citrate de plutonium 239 et qui avaient montré que, sur la base des énergies $\alpha$, le plutonium 239 était 2,5 fois plus toxique que le radium 226 :

$$
40 \mathrm{nCi}=100 \mathrm{nCi} / 2,5 \text {. }
$$

- Charge maximale admissible du poumon : $16 \mathrm{nCi}$.

Cette valeur a été calculée. Elle correspond à l'activité du plutonium 239 qui délivre au poumon, chaque année, la dose maximale admissible pour cet organe : 15 rems (le facteur de qualité des $\alpha$ est, dans le calcul, de 10).

- Pour les autres organes, les charges maximales admissibles ont été calculées sur les mêmes principes que celle du poumon. 
- La plupart des expérimentateurs pensent que la valeur de la charge maximale admissible pulmonaire est légèrement trop élevée. Mais il faut reconnaître que les sensibilités relatives des différentes espèces ne sont pas connues avec précision et que, pour la fixation des limites de sécurité, interviennent des facteurs que l'on ne prend pas en compte dans une expérience menée dans des conditions aussi idéales que possible.

- De toute façon, les conditions réelles de travail entraînent une contamination aérienne très inférieure aux limites maximales admissibles et présentent les meilleures garanties possibles pour la manipulation en toute sécurité du plutonium et des transplutoniens.

\section{SURVEILLANCE DES TRAVAILLEURS}

Les travailleurs sont surveillés en utilisant deux méthodes de mesure : la mesure directe de l'activité pulmonaire pratiquée avec un détecteur à l'extérieur de l'organisme et la mesure indirecte de la contamination interne pratiquées en adaptant l'analyse des excreta à un modèle métabolique.

La détection externe donne des résultats rapides mais a une sensibilité suffisante uniquement pour l'américium 241. Pour les plutonium et le curium, elle ne permet que de diagnostiquer des contaminations accidentelles à niveau relativement élevé.

La mesure indirecte est utilisée pour la surveillance de routine. Elle est pratiquée à partir des urines ou des fèces. Sa sensibilité est suffisante car on sait aujourd'hui, en comparant les résultats de l'estimation basée sur les excreta et les données fournies par des autopsies que l'on peut estimer des contaminations très inférieures aux charges maximales admissibles.

\section{CONCLUSION}

Le plutonium est, de tous les polluants, celui dont la toxicité est la mieux connue. Sa nature chimique le rend incompatible avec le fonctionnement de la matière vivante dans laquelle il se comporte toujours comme un corps étranger et ce que l'on sait aujourd'hui, à partir de l'expérimentation animale, sur la toxicité du plutonium permet d'espérer que les travailleurs qui le manipulent n'auront pas à en souffrir.

\section{BIBLIOGRAPHIE}

[1] BAIR W. J. A radiobiological assessment of the spatial distribution of radiation dose from inhaled plutonium. USAEC report WASH-1320, 1974.

[2] Lafuma J. Diagnostic et traitement d'un cas d'intoxication par le Plutonium In Diagnosis and treatment of radioactive poisoning, Vienna, 15-18 octobre 1962. Vienne, AIEA, 1963.

[3] Lusbaugh C. C. et Langham J. A dermal lesion from implanted plutonium. Arch. Dermatol., 1962, 86, 46. 
[4] Morin M., Nénot J.-C., Masse R., Nolibé D., Metivier H. et Lafuma J. Étude expérimentale de l'action des radio-éléments émetteurs $\alpha$ inhalés. Raccourcissement de la durée de la vie et induction de cancers. Influence de la dose totale, du débit de dose, de l'étalement de la dose dans le temps, de la répartition spatiale de la dose. In Biological and environmental effects of low-level radiation, Chicago, 3-7 novembre 1975. Vienne, AIEA, 1976, 2, 109-119.

[5] Chameaud J., Perraud R., Masse R., Nénot J.-C. et Lafuma J. Cancers du poumon provoqués chez le rat par le radon et ses descendants à diverses concentrations. Comparaison de la relation dose-effet chez l'homme et chez l'animal. In Biological and environmental effects of low-level radiation, Chicago, 3-7 novembre 1975. Vienne, AIEA, 1976, 2, 223-228.

[6] Lafuma J. Les radio-éléments inhalés. Séminaire de la Société française de radioprotection, Paris, 19-20 mars 1974. Radioprotection, 1974, 9, 15-25.

[7] Chameaud J., Perraud R., Lafuma J., Masse R. et Pradel J. Lesions and lung cancers induced in rats by inhaled radon 222 at various equilibriums with radon daughters. In Experimental respiratory carcinogenesis and bioassays, Seattle, June 23-261974 (Karbe E., Parke J.-P., Eds), Berlin, Springer Verlag, 1974, 411-421.

[8] Nolibé D., Masse R., Fritsch P. et Lafuma J. Greffe chez la souris athymique de tissu pulmonaire contaminé par l'oxyde de plutonium. In Biological and environmental effects of low-level radiation, Chicago, 3-7 novembre 1975. Vienne, AIEA, 1976, 2, 95-107. 